\title{
Prices vs Quantities with Multiple Pollutants*
}

\author{
Stefan Ambec ${ }^{\dagger}$ and Jessica Coria ${ }^{\ddagger}$
}

September 26, 2011

\begin{abstract}
We examine the choice of policy instrument price, quantity, or a mix of the two when two pollutants are regulated and firms' abatement costs are private information. A key parameter that affects this choice is the technological externality between the abatement efforts involved, i.e., whether they are substitutes or complements. If they are complements, a mix policy instrument with a tax on one pollutant and a quota on the other is sometime preferable, even if the pollutants are identical in terms of benefits and costs of abatement. Yet, if they are substitutes, the mix policy is dominated by taxes or quotas.
\end{abstract}

Key Words: pollution, environmental regulation, policy mixes, tax, emission standard, asymmetric information.

JEL classification: D62, Q50, Q53, Q58.

\footnotetext{
${ }^{*}$ Research funding from the Adlerbertska Foundation, the Swedish Research Council (FORMAS), the Biodiversity Ecosystems and Climate Change Program (BECC) to the Environmental Economics Unit at the University of Gothenburg, and the French Agence Nationale de la Recherche (ANR) through the project ANR-08-JCJC-0111-01 is gratefully acknowledged.

${ }^{\dagger}$ Toulouse School of Economics (INRA-LERNA) and the University of Gothenburg. Email: stefan.ambec@toulouse.inra.fr.

${ }^{\ddagger}$ University of Gothenburg. Email: Jessica.Coria@economics.gu.se
} 


\section{Introduction}

In 1974, Weitzman wrote one of the most influential papers in environmental economics. It deals with the choice of policy instruments when firms' costs are private information. When applied to pollution mitigation issues, the choice is between a price, or marketbased instrument (such as a tax or tradable emission allowances) and a quantity instrument (such as an emission standard or quota). Weitzman (1974) shows how this choice is driven by two parameters: the slopes of the marginal benefit of reducing pollution and of the firm's abatement costs. A price-based instrument should be preferred when benefits are relatively flat compared to the costs, while quantity-based instruments are better when benefits are steeper than costs.

The analysis by Weitzman concerns the choice of a policy instrument to regulate a single pollutant. However, in practice, production processes are typically accompanied by the emissions of multiple pollutants. Furthermore, abatement technologies can jointly reduce several pollutants at the same time. In some cases, the joint abatement creates some synergies (often referred to as ancillary benefits) while in others cases, certain pollutants are reduced at the cost of increasing others. Thus, pollutants can be considered as complements or substitutes in the abatement process (Burtraw et al. 2003, Bollen et al. 2009, and Holland 2010).

There are examples abound. ${ }^{1}$ For instance, catalytic converters for cars simultaneously reduce emissions of nitrogen oxides (NOx) and volatile organic compounds (VOC) (Bruvoll and Medin 2003), and strategies to reduce nitrogen from sewage plants also reduce phosphorus (Elofsson 2003). In the case of substitutes, recent changes in technology to alleviate the acid deposition problem indicate some disadvantages to the climate change issue: scrubbers installed in power plants neutralise the acids at the expense of

\footnotetext{
${ }^{1}$ It is worth noting that interactions among pollutants might also occur on the damage side. For example, the build-up of ozone (one of the substances for which national air quality standards are most widely violated) results from complicated reactions involving several pollutants in the presence of solar energy. NOx emissions retard ozone formation (Innes 1981 and Repetto, 1987) while methane enhances ozone transport. Therefore, a methane emission reduction strategy would yield the co-benefit of reducing of ozone concentrations while NOx controls would be counterproductive in controlling ozone levels.
} 
increased energy consumption, coal burning and increased carbon dioxide (CO2) emissions (Moslener and Requate 2007, Holland 2010). The increased use of biomass also creates some trade-offs in emissions control. Biomass is helpful in reducing CO2 emissions; however, it also causes increased emissions of particulate matter (PM), carbon monoxide $(\mathrm{CO})$ and VOC. In turn, this means a higher risk to human health for the population exposed to these pollutants (see Syri et al. 2001, Brännlund and Kristöm 2001 and Proost and Van Regemorter 2003 for empirical evidence). ${ }^{2}$

In this paper, we extend Weitzman's Prices vs.Quantities analysis to multiple pollutants. We compare the performance of different policy mixes when firms can reduce the emissions of two pollutants simultaneously through the use of multi-pollutant abatement technologies. We consider two cases. (1) the case where the regulator knows that the abatement technology used by the firm exhibits some complementarity or substitutivity in abatement efforts, but there is asymmetric information on the magnitude of the marginal cost; and (2) the case where the regulator does not know whether abatement efforts are complements or substitutes, which is the only source of asymmetric information. Both cases are relevant in practice. It could be that the properties of the technology used for abating pollution are well known, although the cost of implementing and running this technology is firm specific. Alternatively, abatement might require development or adoption of new technologies for which the externality in abatement cost between the two pollutants is unknown a priori. Also, sometimes there are many technologies available in the market; some could exhibit some complementarity and some could exhibit some substitution. It is the firm's own decision to choose among them. ${ }^{3}$

We study the effects of the choice among only emission taxes, only emission norms or non-tradable quotas, and a policy mix where taxes are used to control the emissions

\footnotetext{
${ }^{2}$ Other examples can be found in agriculture. Farmers can decrease their use of chemical fertilizers (and thus CO2 emissions) by substituting with natural fertilizers from cattle breeding but at a cost of producing more methane. Pesticide can be complements or substitutes to fertilizers depending on the crop and the targeted pests.

${ }^{3}$ For instance, an electricity producer can switch to several sources of energy. Switching from coal to natural gas reduces the emissions of both $\mathrm{NOx}$ and $\mathrm{CO} 2$, while switching from natural gas to biomass potentially reduces $\mathrm{CO} 2$ emissions and increases NOx emissions.
} 
of one pollutant while an emission norm is intended to reduce the emissions of the other. In practice, market-based and quantity policy instruments are used to regulate multi-pollutant firms. For example, in Europe emissions of SO2 and NOx are regulated through taxes and in the U.S. emissions of SO2 are regulated through cap-and-trade while the emissions of several local air pollutants, such as particulate matter or VOC, are mainly controlled through emission standards.

To the best of our knowledge, this is the first study looking at the effects of the choice of policy mixes involving different combinations of price-based and quantity-based regulations in the context of multiple pollutants and asymmetric information. Several papers have analyzed the design of environmental policies with multiple pollutants but under perfect information. In Endres (1985) and Repetto (1987), two pollutants interact in the social damage function, while in our paper they do not. Moslener and Requate (2007) and Kuosman and Laukkanen (2011) deal with multiple pollutant abatement strategies in a dynamic framework. Their focus is on the optimal emission path, and not on the choice of regulation instruments. Climate change mitigation models also consider multi-gas control strategies (see for instance Nordhaus 2000 and Tol 2006) to evaluate emission path scenarios. Montero (2001) and Caplan and Emilson (2005) analyze the design of environmental regulations with several pollutants. Contrary to our paper, they consider only one instrument, namely marketable emission allowances.

Regarding the interaction of policies in the context of a single pollutant, some authors have analyzed the welfare effects of a hybrid policy encompassing both price and quantity mechanisms in Weitzman (1974)'s framework (see for instance Roberts and Spence 1976 and Pizer 2002). Notably, Mandell (2008) shows that with several firms and one pollutant, a mixed scheme consisting of taxing some firms and regulating others through a cap-and-trade system might be more efficient. Similarly, we provide some rationale for a mix policy where one pollutant is taxed while the other is limited by an emission standard.

The paper is organized as follows. In Section 2, we compute the abatement levels and welfare under the three policy instruments mentioned above when the regulator knows whether the pollutants are substitutes or complements, but there is asymmetric 
information regarding the marginal abatement costs. In Section 3, we consider the case when the regulator does not observe whether the pollutants are substitutes or complements. Finally, Section 4 concludes the paper.

\section{Complementarity or substitutivity known by the regu- lator}

A firm is emitting two pollutants: pollutant 1 and pollutant 2 . The pollutants are symmetric in terms of costs for the firm and for society. The cost of reducing emissions by $q_{i}$ units for $i=1,2$ for the firm is

$$
C\left(q_{1}, q_{2}, \omega, \theta\right)=\frac{m q_{1}^{2}}{2}+\frac{m q_{2}^{2}}{2}+\omega q_{1} q_{2}+\theta\left(q_{1}+q_{2}\right)
$$

The parameter $\omega$ captures the complementarity or substitutivity of the two pollutants. If $\omega<0(\omega>0)$, the two pollutants are complements (substitutes) in the cost functions in the sense that reducing pollution of one pollutant decreases (increases) the marginal cost of reducing pollution of the other. The parameters $m$ and $\omega$ are commonknowledge for $i=1,2$. By contrast, following Weitzman (1974), $\theta$ is private information. The regulator believes that $\theta \in[\underline{\theta}, \bar{\theta}]$ and that the expected value of $\theta$ is zero: $E[\theta]=0$.

Let the benefit from reducing emissions of pollutant $i$ be

$$
B\left(q_{i}\right)=a q_{i}-\frac{b}{2} q_{i}^{2} .
$$

Here we also assume symmetry and separability: a reduction of pollutant $i$ does not reduce the damage due to pollutant $j$.

Total welfare with pollution reductions $q_{1}$ and $q_{2}$ is

$$
W\left(q_{1}, q_{2}, \omega, \theta\right)=B\left(q_{1}\right)+B\left(q_{2}\right)-C\left(q_{1}, q_{2}, \omega, \theta\right) .
$$

Consistent with Weitzman (1974), marginal costs and benefits are linear with pollution abatement:

$$
C_{i}\left(q_{1}, q_{2}, \omega, \theta\right)=m q_{i}+\omega q_{j}+\theta
$$


and

$$
B_{i}\left(q_{i}\right)=a-b q_{i}
$$

for $i=1,2$ and $j \neq i$. We assume $m+\omega>0$ and $m+\omega>\theta$ to insure increasing and strictly convex marginal costs. ${ }^{4}$ Similarly, the benefit function is increasing and weakly concave: $B_{i}\left(q_{i}\right)=a-b q_{i}>0$ and $B_{i i}\left(q_{i}\right)=-b \leq 0$ for every $q_{i} \in\left[0, \bar{q}_{i}\right]$ and $i=1,2$.

We first determine the efficient pollution abatement before examining regulations. For a given realisation of $\omega$, the first-best emission abatements $\left(q_{1}^{*}, q_{2}^{*}\right)$ maximise the expost welfare $W\left(q_{1}, q_{2}, \omega, \theta\right)$, which yields the following first-order conditions:

$$
B_{i}\left(q_{i}^{*}\right)=C_{i}\left(q_{i}^{*}, q_{j}^{*}, \omega, \theta\right)
$$

for $i=1,2$ with $j \neq i$. The marginal benefit of each pollutant should be equal to its marginal cost. Since the two pollutants are symmetric, efficient abatement is the same, $q_{1}^{*}=q_{2}^{*}=q^{*}$. Substituting with the functional forms, the above condition simplifies to

$$
a-b q^{*}=(m+\omega) q^{*}+\theta
$$

for $i=1,2$ with $j \neq i$. Therefore, expost efficient abatement levels $q_{1}^{*}=q_{2}^{*}=q^{*}$ are defined $\operatorname{as}^{5}$

$$
q^{*}=\frac{a-\theta}{b+m+\omega} .
$$

The quantity regulation (hereinafter referred to as quota) maximizes expected total welfare $E\left[W\left(q_{1}, q_{2}, \omega, \theta\right)\right]$ with respect to quotas that define minimal abatement levels $\bar{q}_{1}$ and $\bar{q}_{2} \cdot{ }^{6}$ It leads to the first-order condition of equalizing marginal benefit with expected marginal costs:

$$
B_{i}\left(q_{i}\right)=E\left[C_{i}\left(q_{i}, q_{j}, \omega, \theta\right)\right]
$$

\footnotetext{
${ }^{4}$ That is, $C_{i}\left(q_{1}, q_{2}, \omega, \theta\right)>0, \quad C_{i i}\left(q_{1}, q_{2}, \omega, \theta\right)>0$, and $C_{i i}\left(q_{1}, q_{2}, \omega, \theta\right) C_{j j}\left(q_{1}, q_{2}, \omega, \theta\right)-$ $\left[C_{i j}\left(q_{1}, q_{2}, \omega, \theta\right)\right]^{2}>0$ for any $q_{i} \in\left[0, \bar{q}_{i}\right]$ for $i, j=1,2$ and $j \neq i$, where $\bar{q}_{i}$ is an upper bound on abatement (e.g., pollution reduction form laisser-faire). Moslener and Requate (2007) have similar assumptions on the cost function. They imply that the second-order conditions of the maximization programs below hold.

${ }^{5}$ To simplify notation, we write $q^{*}$ instead of $q^{*}(\omega, \theta)$ even though ex post efficient abatements depend on $\omega$ and $\theta$.

${ }^{6}$ Quotas are obviously binding.
} 
for $i=1,2, j \neq i$. With symmetric benefit and costs, the quotas are the same $\bar{q}_{1}=\bar{q}_{2}=\bar{q}$ and the above first-order condition simplifies to

$$
a-b \bar{q}=(m+\omega) \bar{q}
$$

The quotas are therefore

$$
\bar{q}=\frac{a}{b+m+\omega}=q^{*}+\frac{\theta}{b+m+\omega}
$$

for both pollutants. ${ }^{7}$

Under the price regulation (hereinafter referred to as tax), a firm of cost type $\theta$ reacts to the tax rate $\tau_{i}$ for pollutant $i$ by equalising its marginal abatement cost to the tax rate for $i=1,2:^{8}$

$$
\tau_{i}=C_{i}\left(q_{i}, q_{j}, \omega, \theta\right)
$$

for $i=1,2, j \neq i$ and every $\theta \in[\underline{\theta}, \bar{\theta}]$. The regulator maximizes expected welfare $E\left[W\left(q_{1}, q_{2}, \omega, \theta\right)\right]$ subject to the firm's reaction constraints (5) for $i=1,2$. Since pollutants are symmetric, the tax rate is the same $\tau_{1}=\tau_{2}=\tau$ and so are abatement levels under tax $q_{1}^{\tau}=q_{2}^{\tau}=q^{\tau}$. The firm's reaction (or incentive-compatibility) constraints (5) for $i=1,2$ simplify to

$$
\tau=(m+\omega) q^{\tau}+\theta
$$

Maximising $E\left[W\left(q^{\tau}, q^{\tau}, \omega, \theta\right)\right]$ with respect to $\tau$ under the firm's reaction constraint (6) leads the regulator to choose the tax rate corresponding to the marginal benefit at the exante first-best $\tau=a-b \bar{q}$, while the firm reacts by choosing

$$
q^{\tau}=\frac{\tau-\theta}{m+\omega}=\frac{a-\theta}{m+\omega}-\frac{b}{m+\omega} \bar{q}=\frac{a}{b+m+\omega}-\frac{\theta}{m+\omega}=\bar{q}-\frac{\theta}{m+\omega} .
$$

Under the mix policy, one pollutant is regulated with quotas while the other is taxed. The quota $\bar{q}$ is defined as before in (4), while the optimal tax rate is still $\tau=a-b \bar{q}$. The firm reacts by abating to equalise marginal cost to tax:

\footnotetext{
${ }^{7}$ Notice that $\bar{q}=E\left[q^{*}\right]$ as in Weitzman (1974).

${ }^{8}$ To simplify notation, we write $q^{\tau}$ instead of $q^{\tau}(\omega, \theta)$ even though abatements under tax depend on $\omega$ and $\theta$.
} 


$$
\tau=m q+\omega \bar{q}+\theta
$$

Therefore, unlike with only tax, under the mix policy the abatement level for the other pollutant is fixed by the quota, which also constrains the abatement level of the pollutant taxed. The first-order condition (8) leads to the abatement level

$$
\bar{q}^{\tau}=\frac{\tau-\theta}{m}-\frac{\omega}{m} \bar{q}=\frac{a-\theta}{m}-\frac{\omega+b}{m} \bar{q}=\frac{a}{b+m+\omega}-\frac{\theta}{m}=\bar{q}-\frac{\theta}{m} .
$$

Let us summarise our analysis in two pictures. We graph the abatement benefit and abatement cost functions for complements and substitutes (when marginal costs are higher and lower than expected) in Figures 1 and 2, respectively. We denote a positive realisation of $\theta$ by $\theta^{+}$and a negative one by $\theta^{-}$. 
Case $\omega<0$

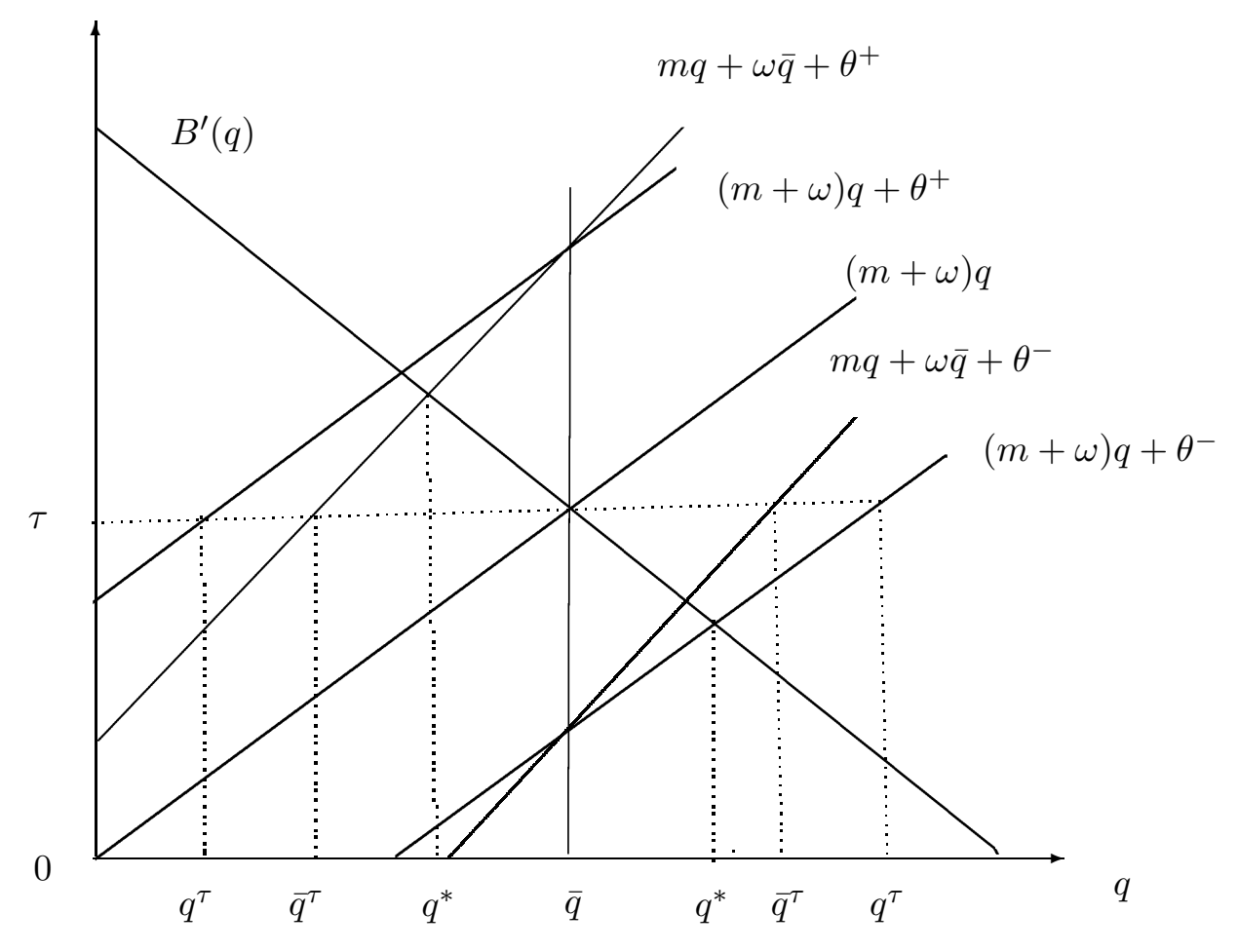

Figure 1. Complement pollutants $\omega<0$ known

In Figure 1, we represent the case where $\omega<0$. The quota level $\bar{q}$ is set where the marginal benefit curve $B^{\prime}(q)=a-b q$ crosses the expected marginal cost curve $(m+\omega) q$. The tax level $\tau$ is equal to the marginal benefit and the expected marginal cost at $\bar{q}$. When the realised cost is lower than expected, i.e. $\theta^{-}$, the realised marginal cost curve under only taxes lies below the expected one with the same slope $(m+\omega)$. The firm then chooses to abate both pollutants up to $q^{\tau}$, where the realised marginal cost under taxes on both pollutants equals the tax level $\tau$.

Under the mix policy, one pollutant is fixed by the quota, and thus the firm's marginal abatement is $m q+\omega \bar{q}+\theta^{-}$. It crosses the expected marginal cost curve $(m+\omega) q$ for an abatement level $\bar{q}$. Moreover, note that since the slope of the expected marginal cost curve is $(m+\omega)$, while the slope of the marginal cost curve under the mix policy is $m$, the latter is steeper for complement pollutants, since $m>m+\omega$ (the reverse 
holds for substitutes as $m<m+\omega$ in such case). Therefore, the abatement level $\bar{q}^{\tau}$ is lower than under the regulation that imposes taxes on both pollutants (reversely, it is higher for substitute pollutants, see Figure 2). ${ }^{9}$ The abatement level under taxes on both pollutants is also larger than under quotas on both pollutants when the realisation of $\theta$ is negative. Since the firm compares the marginal cost to the benefit of abatement, which is the tax level calibrated on an expected higher cost, it finds it profitable to abate further to save taxes.

Hence, with only taxes, both pollutant abatements are adjusted by the firm, while under the mix policy only the taxed pollutant is adjusted - say pollutant 1- while the other, i.e. pollutant 2 , is emitted according to the quota. If the pollutants are complements $(\omega<0$, see Figure 1), the adjustment of pollutant 2 abatement further reduces the abatement cost of pollutant 1 . As a consequence, the firm further increases the abatement of pollutant 1 when pollutant 2 is taxed, in contrast to when it regulated with quotas, and so $\bar{q}^{\tau}<q^{\tau}$. The reverse holds if the pollutants are substitutes $(\omega>0$, see Figure 2). Since the marginal abatement cost of pollutant 1 decreases to a larger extent if pollutant 2 is regulated though a quota, $\bar{q}^{\tau}>q^{\tau}$ in such case.

The case when marginal costs are higher than expected is symmetric. Since $\theta^{+}>0$, the realised marginal cost curve when both pollutants are regulated through taxes lies above the expected one with the same slope $(m+\omega)$. Under the mix policy, the firm's marginal abatement is $m q+\omega \bar{q}+\theta^{+}$. In Figure 1, the slope of the marginal abatement cost under the mix policy is higher than the slope of the expected marginal cost, as pollutants are complements. Hence, there is more abatement under the mix policy than under tax for the taxed pollutant. The reverse holds in Figure 2.

\footnotetext{
${ }^{9}$ Note that if $\omega=0$, then $\bar{q}^{\tau}=q^{\tau}$ since the slope of the marginal cost curve for one pollutant does not depend on the regulation instrument for the other.
} 


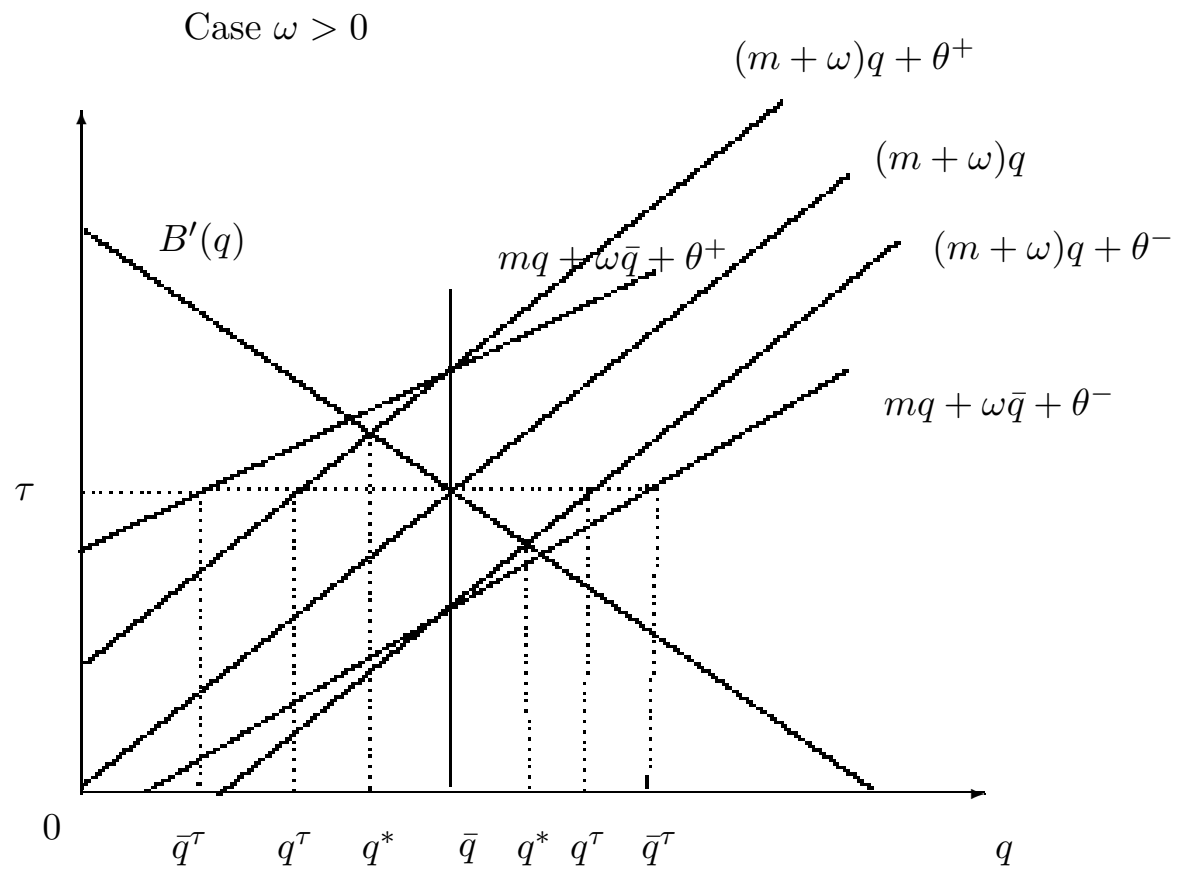

Figure 2: Substitute pollutants $\omega>0$ known

Consistent with Figures 1 and 2, we can rank abatement levels using (4), (7) and (9). It leads to Lemmas 1 and 2.

Lemma 1 Abatement under tax $q^{\tau}$, under quota $\bar{q}$, and under the mix policy for the taxed pollutant $\bar{q}^{\tau}$ are ranked as follows:

(a) If pollutants are complements $\omega<0$ :

- If $b>|\omega|$, then $q^{\tau}<\bar{q}^{\tau}<q^{*}<\bar{q}$ for costs higher than expected and $\bar{q}<$ $q^{*}<\bar{q}^{\tau}<q^{\tau}$ for costs lower than expected.

-If $b<|\omega|$, then $q^{\tau}<q^{*}<\bar{q}^{\tau}<\bar{q}$ for costs higher than expected and $\bar{q}<\bar{q}^{\tau}<q^{*}<$ $q^{\tau}$ for costs lower than expected.

(b) If pollutants are substitutes, then $\omega>0, \bar{q}^{\tau}<q^{\tau}<q^{*}<\bar{q}$ for costs higher than expected and $\bar{q}<q^{*}<q^{\tau}<\bar{q}^{\tau}$ for costs lower than expected.

Note that if pollutants are complements and $b>|\omega|$, the optimal level of abatement lies between $\bar{q}^{\tau}$ and $\bar{q}$ regardless of $\theta$. When $b<|\omega|$, it lies between $\bar{q}^{\tau}$ and $q^{\tau}$. When 
pollutants are substitutes, the mix policy departs further from the optimal level of abatement than mixes involving just one instrument.

Lemma 2 Total abatement is ranked as follows:

(a) If $b>m+\omega$, then $2 \bar{q}>2 q^{*}>\bar{q}^{\tau}+\bar{q}>2 q^{\tau}$ for costs higher than expected and $2 q^{\tau}>\bar{q}^{\tau}+\bar{q}>2 q^{*}>2 \bar{q}$ for costs lower than expected.

(b) If $b<m+\omega$, then $2 \bar{q}>\bar{q}^{\tau}+\bar{q}>2 q^{*}>2 q^{\tau}$ for costs higher than expected and $2 q^{\tau}>2 q^{*}>\bar{q}^{\tau}+\bar{q}>2 \bar{q}$ for costs lower than expected.

Overall the rankings of abatement efforts under only prices and under only quantities are consistent with Weitzman (1974): under-abatement under prices and overabatement under quantities if marginal costs are higher than expected. The reverse holds if costs are lower than expected. Both results hold regardless of $\omega$. Hence, the issue of complementarity vs. substitutivity in abatement costs does not seem to matter when comparing abatement levels under prices and quantities. Nevertheless, as we will see later on, it does affect the comparison of welfare as the marginal cost to be considered now should take into account the joint abatement parameter $m+\omega$ and not only $m$. This is important since it determines the choice of regulation instrument.

What is new compared to Weitzman (1974) is the mix policy whereby one pollutant is taxed and the other is constrained by a quota. In this case, the ranking of abatement for the pollutant that is taxed does depend on $\omega$. To see this, consider first the case where the marginal abatement cost is higher than expected. From Lemma 1, we know that in that case $\bar{q}>q^{\tau}$. Then $m q+\omega \bar{q}>m q+\omega q^{\tau}$ if $\omega>0$ (substitute pollutants) and $m q+\omega \bar{q}<m q+\omega q^{\tau}$ if $\omega<0$ (complement pollutants). When equalising the marginal cost of abating to its marginal benefit $\tau$, the firm abates less under the mix policy in the former than in the latter case. By analogy, $\bar{q}<q^{\tau}$ if the marginal cost is lower than expected. Then $m q+\omega \bar{q}<m q+\omega q^{\tau}$ if $\omega>0$ and $m q+\omega \bar{q}>m q+\omega q^{\tau}$ if $\omega<0$. Therefore, the incentives go in opposite directions: the firm abates more under the mix policy if $\omega>0$ and less if $\omega<0$. Overall, such an abatement strategy departs further from the first-best when pollutants are substitutes, while it moves closer to the first-best 
when pollutants are complements. ${ }^{10}$ It is then no surprise that the mix policy is never a good instrument when pollutants are substitutes whereas it could eventually dominate the price or quantity instruments when they are complements, as we will see latter.

To compare the performance of the three regulations, we need to compute the welfare. This comparison is done in Appendix A, and it leads to the following proposition:

Proposition 1 We have the following ranking of regulations for both the ex ante and ex post welfare for any realisation of $\omega$.

(a) Under substitute pollutants, the quota regulation dominates if $b>m+\omega$; the tax regulation dominates otherwise. The mix regulation is always dominated by quotas or taxes.

(b) Under complement pollutants, the quota regulation dominates if $b>m$, the mix regulation dominates for $m>b>\widehat{b}$ and the tax regulation dominates for $b<\widehat{b}$, where $\widehat{b}$ is defined in (23).

The result (a) is just a generalisation of Weitzman (1974) with multiple pollutants. It basically tells us that the criteria for choosing policy instruments under uncertainty is the same as in Weitzman (1974), yet the marginal cost to be considered should take into account the joint abatement parameter $\omega$ and not only $m$. The result (b) is more interesting. Compared to Weitzman (1974), having more than one pollutant enlarges the set of instruments: some pollutants can be priced while others can be regulated by quotas. The proposition points out that a mix policy can be a good option in some cases, even though the pollutants are symmetric in term of costs and benefit, and hence, one would expect that the same instrument should be applied to both pollutants. Indeed, the policy mix is welfare enhancing when pollutants are complements and the slope of the marginal benefit and cost curves are close. In contrast, for very different marginal cost and benefit slopes, only one instrument should be applied to both pollutants, in the spirit of Weitzman.

\footnotetext{
${ }^{10}$ In the latter case, whether it is higher or lower than the first-best depends on the magnitudes of $b$ and $\omega$.
} 


\section{Complementarity or substitutivity unknown by the reg- ulator}

Suppose now that the regulator does not know whether the two pollutants are complements or substitutes in the firm's cost abatement function. Now the complementarity and substitutivity parameter $\omega$ is subject to information asymmetry, and we set $\theta=0$. The cost function is simplified to

$$
C\left(q_{1}, q_{2}, \omega\right)=\frac{m q_{1}^{2}}{2}+\frac{m q_{2}^{2}}{2}+\omega q_{1} q_{2}
$$

where $\omega$ is the firm's private information. The regulator believes that $\omega \in[\underline{\omega}, \bar{\omega}]$ and $E[\omega]=0$. The parameter $m$ is common knowledge.

The benefit of pollution abatement is the same as defined by (1) for $i=1,2$. Total welfare with pollution reductions $q_{1}$ and $q_{2}$ is then

$$
W\left(q_{1}, q_{2}, \omega\right)=B\left(q_{1}\right)+B\left(q_{2}\right)-C\left(q_{1}, q_{2}, \omega\right) .
$$

Marginal benefits and costs are

$$
\begin{aligned}
& B_{i}\left(q_{i}\right)=a-b q_{i}, \\
& C_{i}\left(q_{1}, q_{2}, \omega\right)=m q_{i}+\omega q_{j},
\end{aligned}
$$

for $i=1,2$ and $j \neq i$. For a given realisation of $\omega$, the first-best emission abatements $\left(q_{1}^{*}, q_{2}^{*}\right)$ maximizes the ex-post welfare $W\left(q_{1}, q_{2}, \omega\right)$ which yields the following first order conditions:

$$
B_{i}\left(q_{i}^{*}\right)=C_{i}\left(q_{i}^{*}, q_{j}^{*}, \omega\right)
$$

for $i=1,2$ with $j \neq i$. Since the two pollutants are symmetric, efficient abatement is the same $q_{1}^{*}=q_{2}^{*}=q^{*}$. Substituting with the functional forms, the above condition simplifies to

$$
a-b q^{*}=(m+\omega) q^{*}
$$


Therefore, expost efficient abatement levels are

$$
q^{*}=\frac{a}{b+m+\omega}
$$

for both pollutants. ${ }^{11}$

The quota regulation equalises marginal benefit to expected marginal costs:

$$
B_{i}\left(q_{i}\right)=E\left[C_{i}\left(q_{i}, q_{j}, \omega\right)\right],
$$

for $i=1,2, j \neq i$. Since the benefit and costs are symmetric, quotas are the same, $\bar{q}_{1}=\bar{q}_{2}=\bar{q}$, and the above first-order condition simplifies to

$$
a-b \bar{q}=m \bar{q} .
$$

Thus, the quotas for both pollutants are

$$
\bar{q}=\frac{a}{b+m} .
$$

Under the tax regulation, a firm of cost type $\omega$ reacts to the tax rate $\tau_{i}$ for pollutant $i$ by equalising its marginal abatement cost to the tax rate for $i=1,2:^{12}$

$$
\tau_{i}=C_{i}\left(q_{i}, q_{j}, \omega\right)
$$

for $i=1,2, j \neq i$ and every $\omega \in[\underline{\omega}, \bar{\omega}]$. The regulator maximises the expected welfare $E\left[W\left(q_{1}, q_{2}, \omega\right)\right]$ subject to the firm's reaction constraints (14) for $i=1,2$. Since pollutants are symmetric, the tax rate is the same, $\tau_{1}=\tau_{2}=\tau$, and so are abatement levels under tax, $q_{1}^{\tau}=q_{2}^{\tau}=q^{\tau}$. The firm's reaction (or incentive-compatibility) constraints (14) for $i=1,2$ simplify to

$$
\tau=(m+\omega) q^{\tau}
$$

Maximising $E\left[W\left(q^{\tau}, q^{\tau}\right)\right]$ with respect to $\tau$ under the firm's reaction constraints (15) leads the regulator to choose the tax rates corresponding to the marginal benefit at the exante first-best $\tau=a-b \bar{q}$, while the firm reacts by choosing

$$
q^{\tau}=\frac{\tau}{m+\omega}=\frac{a-b \bar{q}}{m+\omega}=\frac{m}{m+\omega} \bar{q}=\frac{a m}{(m+\omega)(b+m)},
$$

\footnotetext{
${ }^{11}$ To simplify notation, we write $q^{*}$ instead of $q^{*}(\omega)$ even though ex post efficient abatements depend on $\omega$.

${ }^{12}$ To simplify notation, we write $q^{\tau}$ instead of $q^{\tau}(\omega)$ even though abatements under tax depend on $\omega$.
} 
where the last two equalities come from the definition of $\bar{q}$ in (13).

Consider now the mix policy consisting of setting a tax for pollutant 1 and a quota for pollutant 2. The best policy mix $(\tau, \bar{q})$ maximises the expected welfare $E[W(\bar{q}, q)]$ under the firm's reaction constraint:

$$
\tau=m q+\omega \bar{q}
$$

Hence, as in the preview section, and contrary to the tax regulation, with a mix policy, the firm adjusts emissions of one pollutant only, while the other is fixed by the quotas. Consequently, the two reaction constraints (15) and (17) differ. Under our assumption, it is easy to show that the optimal tax-quota policy equalises the marginal benefit to the expected marginal cost for the quota, i.e.

$$
a-b \bar{q}=m \bar{q},
$$

and the tax rate equals the expected marginal cost with a quota or, equivalently, the marginal benefit with a quota:

$$
\tau=a-b \bar{q}=m q .
$$

Hence, firms react to the above tax rate by abating:

$$
\bar{q}^{\tau}=\frac{\tau-\omega \bar{q}}{m}=\frac{m-\omega}{m} \bar{q}=\frac{a(m-\omega)}{m(b+m)} .
$$

The above analysis can more easily be understood using a graph. 


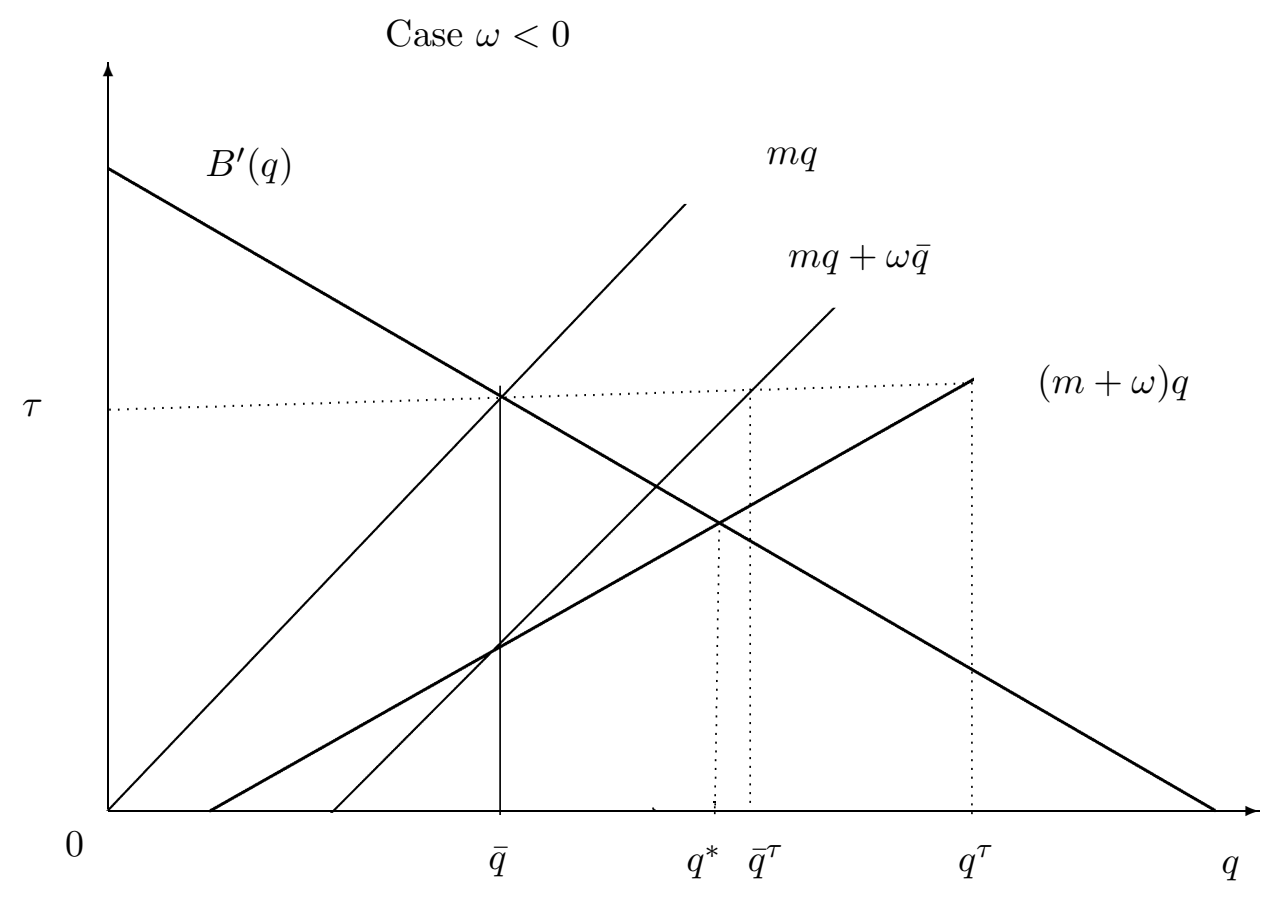

Figure 3. Complement pollutants $\omega<0$ unknown

Figure 3 describes the case when pollutants are complements. The expected marginal abatement cost function is given by $m q$. The realised marginal costs are $m q+\omega \bar{q}$ and $(m+\omega) q$ with the mix policy and with taxes respectively. Hence, the quota $\bar{q}$ and tax rate $\tau$ are found where the marginal benefit curve $B(q)=a-b q$ crosses the expected marginal abatement cost curve. In particular, the tax rate $\tau$ is set to equalise marginal benefit to expected marginal abatement cost.

When abatement efforts turn out to be complements, abatement costs are lower than expected. The firm then chooses to abate more under tax than under quota. Indeed, since the firm compares the marginal cost to the benefit of abatement, which is the tax level calibrated on an expected higher cost, it finds it profitable to abate further to save on taxes. In the graph, the firm picks the abatement level where the tax rate $\tau$ crosses the marginal cost curve $m q+\omega \bar{q}$ or $(m+\omega) q$ depending on whether the other pollutant is regulated with a quota or a tax. Abatement is increased for only one pollutant if the other is regulated with quotas and both if both pollutants are regulated through 
taxes. In the latter case, more abatement of pollutant 2 further reduces the marginal cost of abatement of pollutant 1. Therefore, pollution reduction is increased further under taxes on both pollutants. Formally, if $\omega<0$ then $\bar{q}^{\tau}<q^{\tau}$. This effect is due to the complementarity of abatement effort: if the change were on the level of the marginal cost for pollutant 1 like in Weitzman (1974), then the abatement effort for pollutant 1 would be the same regardless of whether pollutant 2 is regulated through a tax or a quota.

The symmetric case of substitute pollutants $\omega>0$ is represented in Figure 4. 


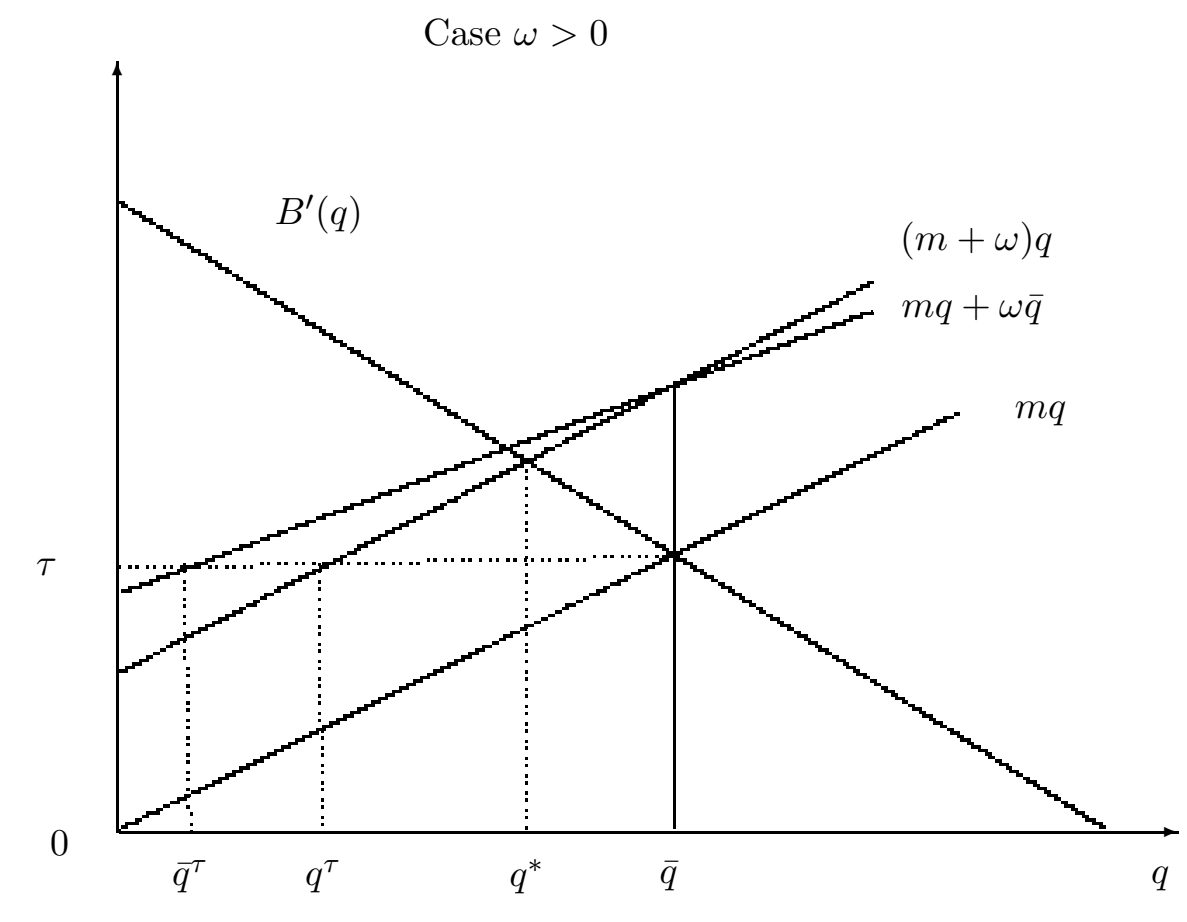

Figure 4. Substitute pollutants $\omega>0$ unknown

In this case, given the tax rate $\tau$, the firm finds it profitable to reduce pollution abatement (or to increase pollution emissions) under tax compared to under quota. Only pollutant 1's emissions abatement is reduced if pollutant 2 is regulated through a quota. Both pollutant abatement are reduced when both are taxed. In the latter case, a decrease in abatement of pollutant 2 reduces the marginal cost of abatement of pollutant 1. As a consequence, the firm increases abatement of pollutant 1 to a larger extent when the pollutant is taxed: $\bar{q}^{\tau}<q^{\tau}$. Therefore, regardless of whether the pollutants are substitutes or complements in the cost function, abatement is higher if the other pollutant is regulated through taxes.

Next we will compare the impact of the three policy instruments on abatement for a given realisation of $\omega$. We will then discuss their impact on expost and exante welfare. Since $m>\omega$ for every $\omega \in[\underline{\omega}, \bar{\omega}]$, comparing equations (13), (16) and (18) for a given $\omega$ leads to Lemmas 3 and 4.

Lemma 3 Abatement under tax $q^{\tau}$, under quota $\bar{q}$, and under the mix policy for the 
taxed pollutant $\bar{q}^{\tau}$ are ranked as follows:

(a) If pollutants are substitutes, $\omega>0, \bar{q}^{\tau}<q^{\tau}<q^{*}<\bar{q}$.

(b) If pollutants are complements, $\omega<0, \bar{q}<q^{*}<\bar{q}^{\tau}<q^{\tau}$.

Lemma 4 Total abatement is ranked as follows:

(a) If pollutants are substitutes, $\omega>0,2 q^{\tau}<\bar{q}^{\tau}+\bar{q}<2 q^{*}<2 \bar{q}$.

(b) If pollutants are complements, $\omega<0,2 \bar{q}<\bar{q}^{\tau}+\bar{q}<2 q^{*}<2 q^{\tau}$.

As in the previous section, Lemma 3 indicates that when pollutants are complements, the mix policy brings the abatement level induced by the taxed pollutant closer to the first-best. Yet when the pollutants are substitutes, the policy mix departs further from the first-best. This can be explained by the interaction of two factors: (1) the technological externality that leads taxes to induce over-abatement when the pollutants are complements, while the reverse holds when they are substitutes; $(2)$ the level of the quota, i.e. if pollutants are complements, the level of abatement under the quota is lower than the first-best, while the reverse holds when they are substitutes. Hence, to anchor the tax to a quota reduces the deviation from the first-best since these two factors set against each other. Instead, these two factors boost each other when pollutants are substitutes; the fact that there is too much abatement of the pollutant subjected to the quota implies that firms must reduce the abatement of the taxed pollutant to a much larger extent than optimal.

Nevertheless, the mix still brings aggregate abatement closer to the optimal. Indeed, as stated by Lemma 4, if the pollutants are substitutes, the optimal level of aggregate abatement $2 q^{*}$ lies between $\bar{q}^{\tau}+\bar{q}$ and $2 \bar{q}$. Under the mix policy, firms abate less than optimal. The reverse holds for the quota regulation. If the pollutants are complements, the optimal level of aggregate abatement $2 q^{*}$ lies between $\bar{q}^{\tau}+\bar{q}$ and $2 q^{\tau}$. Firms abate less than optimal under the mix policy, while there is too much abatement under the tax regulation.

In order to find the best instruments depending on the economic environment, we need to compare welfare under the three regulatory instruments. Let us first consider the 
expost welfare, i.e. for a given realisation of $\omega$. This comparison laid out in Appendix B leads to the following conclusions:

Proposition 2 For a given $\omega$, we have the following results:

(a) The price regulation dominates the quantity regulation if and only if $b<m+\omega$.

(b) The mix policy (tax-quota) dominates the quantity regulation if and only if $b<m$.

Although Proposition 2 does not rank all instruments regarding ex post welfare, it does provide some partial comparisons. Result (a) has the same flavor as the generalisation of Weitzman (1974) in Proposition 1. It turns out that price regulations dominate quota regulations expost when the slope of the marginal benefit functions $b$ is steeper than the slope of the overall marginal costs $m+\omega$ for any realisation of $\omega$. This expost dominance is not easy to translate exante because the slope of the marginal costs $m+\omega$ depends itself on the realisation of $\omega$. Thus, taxes might dominate for some realisation of $\omega$ while quota might dominate for others. We can only conclude that the exante welfare is higher with tax (resp. quota) than with quota (resp. tax) if the marginal benefit functions are steeper that the marginal cost functions for any realisation of $\omega$. This formally leads to (a) and (b) in Corollary 1. From Proposition 2 we can straightforwardly conclude (c) in Corollary 1.

\section{Corollary 1}

(a) Prices dominate quantities if $b<m+\underline{\omega}$.

(b) Quantities dominate prices if $b>m+\bar{\omega}$.

(c) If $b<m$ then at least one pollutant should be taxed.

To be able to fully rank the three instruments, we make a further simplification assumption. We assume that $\omega$ can take only two values $-\delta$ and $+\delta$ with the same probability (so that the $E[\omega]=0$ ) with $m>-\delta$ and $\delta>0$. By comparing exante welfare with the three instruments in Appendix B, we obtain the following ranking:

Proposition 3 If $\omega \in\{-\delta,+\delta\}$ then there exists $\widetilde{b}<m$ such that: 
(a) For $b>m$, the quota regulation dominates.

(b) For $\widetilde{b}<b<m$, the mix regulation dominates.

(c) For $b<\widetilde{b}$, the tax regulation dominates.

When the technological externality parameter $\omega$ is unknown by the regulator, each of the three regulatory instruments can be optimal depending on the slope of the marginal benefit and cost functions. When the marginal benefit function is steeper than the marginal cost function, i.e. when $b>m$, then both pollutants should be regulated with quotas. The intuition is similar to that in Weitzman (1974). With steeper marginal benefit than marginal cost, the loss of welfare is lower with quotas than with taxes since the quotas are closer to the first-best abatement levels than the firm's abatement choices with taxes. Now consider the reverse case $b<m$. Following Weitzman (1974), the tax regulation should dominate the quota regulation. However, with two pollutants, a third instrument, i.e the policy mix (tax on one pollutant and quota on the other) enters into the picture. It indeed dominates taxes when the slope of the marginal cost and benefit curves $b$ and $m$ are close but still $b<m$. This is easy to understand if we compare the firm's abatement strategy under the policy mix and under taxes. Under the policy mix, only one pollutant is chosen by the firm (the other one is fixed by the quota) whereas under taxes both are chosen. If the two pollutants turn out to be complements, then abatement is cheaper for the firm than expected by the regulator. Then, the firm would choose to abate more of each pollutant than under quotas. This is true for only one pollutant under the policy mix and for both pollutants under taxes. Since the increase in abatement of one pollutant further reduces the abatement marginal cost of the other pollutant, the firm abates even more of the taxed pollutant under tax than under the policy mix. The case of substitute pollutants is the oppossite. Since marginal cost is higher than expected, the firm under-abates more of the taxed pollutant under the mix policy than under taxes. The deviation from the first-best for the taxed pollutant under the policy mix is indeed amplified by the technological externality. Yet it is at the benefit of having more abatement of the pollutant regulated by quota When $b$ is close to $m$, more precisely as long as $\widetilde{b}<b<m$, the welfare gain due to the quota of one 
pollutant is higher than the distortion in the level of abatement of the pollutant that is taxed. Therefore the mix policy dominates. Nevertheless, the loss of welfare is higher when $b<\widetilde{b}$ and, therefore, taxes are better.

\section{Conclusion}

In this paper we analyse the effects of the choice of policy instruments when firms can abate more than one pollutant at the same time. We consider successively two cases: (1) the case where the regulator knows that the abatement technology used by the firm exhibit some complementarity or substitutivity in abatement efforts and only the information on the magnitude of the marginal cost is asymmetric; (2) the case where asymmetric information concerns whether or not the pollutants are complement or substitutes in the abatement process.

Overall, the paper highlights that the technological externality parameter is an important component of the choice of instruments under asymmetric information on abatement costs. First, since it impacts marginal costs, it is a key parameter that determines whether pricing both pollutants dominates setting quotas on emissions. When this parameter is known by the regulator, the technological externality plays the same role as in Weitzman (1974). When it is not known by the regulator, then the dominance is not clear since it depends on the distribution of the technological externality parameter. Yet in Proposition 3 we derive conditions on the economic environment for which price or quantity regulations dominate. Second, we provide some rationale for a mix policy instrument consisting of taxing one pollutant and setting a quota on the other. It turns out that this policy mix is optimal when pollutants are complements and the slope of the marginal benefits and costs are close. When the complementarity or substitutability parameter is unknown by the regulator, the mix policy might still dominate for similar slopes for the marginal benefit and cost functions. In this case, all three instruments might dominate depending on marginal costs and benefits.

In our setup with perfectly symmetric pollutants, the dominance of the mix policy is somewhat surprising. Although the two pollutants have exactly the same benefit 
and cost functions, it turn out that they should be regulated differently: one should be taxed and one should be limited with a quota. Intuitively, such a mix policy is attractive because it allows some, but not too much flexibility for the firms. However, whether or not the policy mix brings abatement closer to the first-best depends on whether pollutants are complements or substitutes in the abatement efforts. If pollutants are complements, a regulation based on only taxes leads the firm to too much abatement since it will overlook the abatement costs. The mix policy limits the distortion on the taxed pollutant since the other pollutant is fixed by the quota to a lower level. If they instead are substitutes, there is too much abatement of the pollutant subjected to the quota, which implies that the deviation from the first-best is increased as the firm must reduce the abatement of the pollutant that is taxed to a much larger extent than optimal.

We have assumed that pollutants are symmetric in order to facilitate our presentation. If pollutants were asymmetric, it would be difficult to compare welfare under different policy mixes. However, the rankings of abatement under different policy mixes would remain the same for interior solutions, since the driving force explaining our results is the technological interaction between the pollutants and because of the fact that the regulator can reduce the deviations from the first-best by mixing taxes and quotas.

It should be noted that we have considered that the two pollutants are regulated by a single regulator. Yet, in many contexts, several administrations at different levels (local, national, supranational) deal with different (air, water, waste) pollution problems for historical or practical reasons. We could easily extend our analysis with two regulators, each of them being in charge of one pollutant. Then further sources of inefficiency might arise as they do not coordinate their choices of policy instruments. Such coordination inefficiencies would potentially modify the choice of instruments for a given set of parameters. Although the choice of policy instruments by different regulators is relevant in practice, its analysis is beyond the scope of our paper. 


\subsection{Appendix A}

We first compute the ex post welfare under the three regulations when $\omega$ is known for any realisation of $\theta$. We then rank the three regulations according to the ex post welfare.

$$
\begin{aligned}
& W(\bar{q}, \bar{q}, \omega, \theta)=2 \bar{q}\left(a-\frac{b+m+\omega}{2} \bar{q}-\theta\right)=2 w(\bar{q}, \omega, \theta), \\
& W\left(q^{\tau}, q^{\tau}, \omega, \theta\right)=2 q^{\tau}\left(a-\frac{b+m+\omega}{2} q^{\tau}-\theta\right)=2 w\left(q^{\tau}, \omega, \theta\right), \\
& W\left(\bar{q}^{\tau}, \bar{q}, \omega, \theta\right)=w\left(\bar{q}^{\tau}, \omega, \theta\right)+w(\bar{q}, \omega, \theta),
\end{aligned}
$$

where $w(q, \omega, \theta)=q\left(a-\theta-\frac{b+m+\omega}{2} q\right)$.

\section{Taxes vs quotas}

$$
\begin{aligned}
\Delta W_{1} & =W\left(q^{\tau}, q^{\tau}, \omega, \theta\right)-W(\bar{q}, \bar{q}, \omega, \theta), \\
\Delta W_{1} & =\left(q^{\tau}-\bar{q}\right)\left[2(a-\theta)-(b+m+\omega)\left(q^{\tau}+\bar{q}\right)\right] .
\end{aligned}
$$

Since $q^{\tau}=\frac{m-\omega}{m} \bar{q}$ by $(7)$,

$$
\Delta W_{1}=-\frac{\theta^{2}(b-m-\omega)}{(m+\omega)^{2}} .
$$

Therefore $\Delta W_{1}>0$ if and only if $b<m+\omega$ : taxes dominate quotas if $b<m+\omega$; the reverse holds if $b>m+\omega$.

\section{Mix policy vs quotas}

$$
\begin{aligned}
\Delta W_{2} & =W\left(\bar{q}_{1}^{\tau}, \bar{q}_{2}, \omega, \theta\right)-W\left(\bar{q}_{1}, \bar{q}_{2}, \omega, \theta\right), \\
\Delta W_{2} & =\left[\bar{q}^{\tau}-\bar{q}\right]\left[a-\theta-\frac{b+m}{2}\left(\bar{q}^{\tau}+\bar{q}\right)-\omega \bar{q}\right] .
\end{aligned}
$$

Since $\bar{q}^{\tau}=\bar{q}-\frac{\theta}{m}$ by (9) and using (4), we obtain

$$
\Delta W_{2}=-\frac{\theta^{2}(b-m)}{2 m^{2}} \text {. }
$$

Therefore $\Delta W_{2}>0$ if and only if $b<m$ : the mix policy dominates quotas when $b>m$; the reverse holds when $b<m$. 


\section{Taxes vs mix policy}

$$
\begin{aligned}
\Delta W_{3} & =W\left(q^{\tau}, q^{\tau}, \omega, \theta\right)-W\left(\bar{q}^{\tau}, \bar{q}, \omega, \theta\right)=\Delta W_{1}-\Delta W_{2}, \\
\Delta W_{3} & =\frac{-\theta(b-m-\omega)}{(m+\omega)^{2}}+\frac{\theta^{2}(b-m)}{2 m^{2}} \\
\Delta W_{3} & =\theta^{2} \frac{(b-m)\left(\omega^{2}+2 m \omega-m^{2}\right)+2 m^{2} \omega}{2 m^{2}(m+\omega)^{2}} .
\end{aligned}
$$

Therefore $\Delta W_{3}>0$ if and only if $b<\hat{b}$ where $\hat{b}$ is defined by

$$
\hat{b}=\frac{m\left(m^{2}-\omega^{2}\right)}{m^{2}-2 m \omega-\omega^{2}} .
$$

The tax regulation dominates the mix regulation if $b<\hat{b}$ while the reverse holds if $b>\hat{b}$.

\section{Policy ranking}

- Substitute pollutants $\omega>0$. Since $m<m+\omega$, then quotas dominate for $b>m+\omega$ and taxes dominate for $b<m+\omega$. The mix policy is always dominated by either taxes or quotas.

- Complement pollutants $\omega<0$. Since $m+\omega<m$ and $\hat{b}<b$, each of the three regulations can be the best one: quotas for $b>m$, mix for $b \in[\hat{b}, m]$ and taxes for $b<\hat{b}$.

Since the above ranking holds expost for any realization of $\theta$, then it also holds exante (i.e. with the exante welfare as the regulator's objective).

\subsection{Appendix B}

We first compute the ex post and ex ante welfares under the three regulations when $\omega$ is firm's private information.

$$
W\left(\bar{q}_{1}, \bar{q}_{2}, \omega\right)=\bar{q}[2 a-[b+m+\omega] \bar{q}]
$$




\section{Taxes vs. quotas}

$$
\begin{aligned}
\Delta W_{1} & =W\left(q^{\tau}, q^{\tau}, \omega\right)-W(\bar{q}, \bar{q}, \omega), \\
\Delta W_{1} & =2 a\left[q^{\tau}-\bar{q}\right]-[b+m+\omega]\left[\left(q^{\tau}\right)-(\bar{q})\right], \\
\Delta W_{1} & =\left[q^{\tau}-\bar{q}\right]\left[2 a-[b+m+\omega]\left[q^{\tau}+\bar{q}\right]\right] .
\end{aligned}
$$

Since $q^{\tau}=\frac{m}{m+\omega} \bar{q}$, the last equation becomes

$$
\begin{aligned}
\Delta W_{1} & =a\left[q^{\tau}-\bar{q}\right]\left[2-\frac{[b+m+\omega][2 m+\omega]}{[b+m][m+\omega]}\right], \\
\Delta W_{1} & =-\omega^{2} \bar{q}^{2}\left[\frac{[b-m-\omega]}{[m+\omega]^{2}}\right] .
\end{aligned}
$$

Hence $\Delta W_{1}>0$ if and only if $b<m+\omega$, regardless of $\omega$.

We will now compare the ex ante welfare when $\omega$ can take two values $-\delta$ and $\delta$ with equal probability.

$$
\begin{aligned}
& E\left[\Delta W_{1}\right]=\frac{-\delta^{2} \bar{q}^{2}}{2}\left[\frac{[b-m-\delta]}{[m+\delta]^{2}}+\frac{[b-m+\delta]}{[m-\delta]^{2}}\right], \\
& E\left[\Delta W_{1}\right]=\frac{-\delta^{2} \bar{q}^{2}}{2}\left[\frac{2 b\left[m^{2}+\delta^{2}\right]-2 m\left[m^{2}-\delta^{2}\right]}{[m+\delta]^{2}[m-\delta]^{2}}\right] .
\end{aligned}
$$

In this case, there is a critical value $b^{\prime}=\frac{m\left[m^{2}-\delta^{2}\right]}{m^{2}+\delta^{2}}<m$ determining whether $E\left[\Delta W_{1}\right]$ is positive or negative. If $\left.b<b^{\prime}, E\left[\Delta W_{1}\right)>0\right]$, therefore taxes dominate quotas.

\section{Policy mix vs. quotas}

$$
\begin{aligned}
\Delta W_{2} & =W\left(\bar{q}^{\tau}, \bar{q}, \omega\right)-W(\bar{q}, \bar{q}, \omega), \\
\Delta W_{2} & =\left[\bar{q}^{\tau}-\bar{q}\right]\left[a-\left[\frac{b+m}{2}\right]\left[\bar{q}^{\tau}+\bar{q}\right]-\omega \bar{q}\right] .
\end{aligned}
$$

Since $\bar{q}^{\tau}=\frac{m-\omega}{m} \bar{q}$, the last equality becomes

$$
\Delta W_{2}=-\omega^{2} \bar{q}^{2}\left[\frac{b-m}{2 m^{2}}\right] .
$$

Hence $\Delta W_{2}>0$ if $b<m$, regardless of $\omega$. Therefore the ex ante welfare is higher under the policy mix than under quotas if and only if $b<m$. 


\section{Taxes vs. policy mix}

$$
\begin{aligned}
\Delta W_{3} & =W\left(q^{\tau}, q^{\tau}, \omega\right)-W\left(\bar{q}^{\tau}, \bar{q}, \omega\right)=\Delta W_{1}-\Delta W_{2}, \\
\Delta W_{3} & =a \omega\left[q^{\tau}-\bar{q}\right]\left[\frac{[b-m-\omega]}{[b+m][m+\omega]}\right]-a \omega\left[\bar{q}_{1}^{\tau}-\bar{q}\right]\left[\frac{b-m}{2 m[b+m]}\right], \\
\Delta W_{3} & =w^{2} \bar{q}^{2}\left[\frac{[m-b]\left[m^{2}-\omega^{2}\right]+2 b m \omega}{2 m^{2}[m+\omega]^{2}}\right] .
\end{aligned}
$$

Again, let us consider that $\omega$ takes two values $-\delta$ and $\delta$ with equal probability. The expected variation of welfare is

$$
E\left[\Delta W_{3}\right]=\frac{\delta^{2} \bar{q}^{2}}{2}\left[\frac{[m-b]\left[m^{4}-\delta^{4}\right]-4 b m^{2} \delta^{2}}{m^{2}[m+\delta]^{2}[m-\delta]^{2}}\right]
$$

In this case, there is a critical value $\tilde{b}=\frac{m\left[m^{4}-\delta^{4}\right]}{\left[m^{4}-\delta^{4}\right]+4 m^{2} \delta^{2}}<m$ determining whether $E\left[\Delta W_{3}\right]$ is positive or negative. $E\left[\Delta W_{3}\right]>0$, and therefore taxes dominate the policy mix if and only if $b<\tilde{b}$.

Policy ranking To fully rank the three policy instruments when $\omega$ takes two values with equal probability, note that $m>\tilde{b}>b^{\prime}$.

- If $b>m$, quantities dominate prices and the tax-quantity mix.

- If $b \in[\widetilde{b}, m]$, the policy mix dominates quantities and prices.

- If $b<\widetilde{b}$, prices dominate quantities and the tax-quantity mix. 


\section{References}

[1] Brännlund, R and B. Kriström. 2001. Too hot to handle? Benefits and costs of stimulating the use of biofuels in the Swedish heating sector. Resource and Energy Economics 23(4): 343-358.

[2] Bollen, J., B. van der Zwaan, C. Brink and H. Eerens. 2009. Local air pollution and global climate change: A combined cost-benefit analysis. Resource and Energy Economics 31(3): 161-181.

[3] Bruvoll, A. and H. Medin. 2003. Factors Behind the Environmental Kuznets Curve. A Decomposition of the Changes in Air Pollution. Environmental and Resource Economics 24(1): 27-48.

[4] Burtraw, D., A. Krupnick, K. Palmer, A. Paul, M. Toman, C. Bloyd. 2003. Ancillary benefits of reduced air pollution in the U.S. from moderate greenhouse gas mitigation policies in the electricity sector. Journal of Environmental Economics and Management 45 (3): 650-673.

[5] Caplan, A. and S. Emilson. 2005. An efficient mechanism to control correlated externalities: redistributive transfers and the coexistence of regional and global pollution permit markets. Journal of Environmental Economics and Management 49(1): $68-82$.

[6] Elofsson, K. 2003. Cost-effective reductions of stochastic agricultural loads to the Baltic Sea. Ecological Economics 47(1): 13-31.

[7] Endres, A. 1985. Environmental policy with pollutant interaction, in Pethig, R. (ed.), Public Goods and Public Allocation Policy, Frankfurt/Main, pp. 165-199.

[8] Innes, W.B. 1981. Effect of nitrogen oxide emissions on ozone levels in metropolitan regions. Environmental Science and Technology 15(8): 904-911.

[9] Leightner, J. 1999. Weather-induced changes in the tradeoff between SO2 and NOx at large power plants. Energy Economics 21(3): 239-259.

[10] Holland, S.P. 2010. Spillovers from Climate Policy. NBER Working Paper. 
[11] Kuosman, T. and M. Laukkanen. 2011. (In)Efficient Environmental Policy with Interacting Pollutants. Environmental and Resource Economics 48(4): 629-649.

[12] Mandell, S. 2008. Optimal mix of emissions taxes and cap-and-trade. Journal of Environmental Economics and Management 56(2): 131-140.

[13] Moslener, U. and T. Requate. 2007. Optimal abatement in dynamic multi-pollutant problems when pollutants can be complements or substitutes. Journal of Economic Dynamics and Control 31(7): 2293-2316.

[14] Montero, J.P. 2001. Multipollutant Markets. The RAND Journal of Economics $32(4): 762-774$

[15] Nordhaus, W.D. 2000. Warming the World, Economic Models of Global Warming. MIT Press, Cambridge.

[16] Pizer, W.A. 2002. Combining price and quantity controls to mitigate global climate change. Journal of Public Economics 85(3): 409-434.

[17] Proost Stef and D. Van Regemorter. 2003. Interaction between local air pollution and global warming and its policy implications for Belgium. International Journal of Global Environmental Issues 3 (3): 266-286.

[18] Repetto, R. 1987. The Policy Implications of Non-Convex Environmental Damages: A Smog Control Case Study. Journal of Environmental Economics and Management 14(1): 13-29.

[19] Roberts, M.J. and M. Spence. 1976. Effluent charges and licences under uncertainty. Journal of Public Economics 5(3-4): 193-208.

[20] Syri, S., M. Amann, P. Capros, L. Mantzos, J. Cofala and Z. Klimont. 2001. Low-CO2 energy pathways and regional air pollution in Europe. Energy Policy 29(11): 871-884.

[21] Tol, R. 2006. Multi-gas emissiom reduction for climate change policy: an application of fund. Energy Policy 28(3): 235-250.

[22] Weitzman, M. 1974. Prices vs. Quantities. The Review of Economic Studies XLI: 477-449. 